\title{
EFEITO DO TIPO DE PODA NA PRODUÇÃO E QUALIDADE DA VIDEIRA CV. VÊNUS
}

\section{DIFFERENTS PRUNE EFECTIN VINEGRAPES CV. VÊNUS PRODUCTION AND QUALIT}

\author{
Marcello SOZIM 1 \\ Ricardo Antonio AYUB ${ }^{2}$ \\ Marcelo Barbosa MALGARIM³.
}

\section{RESUMO}

Este experimento foi realizado num vinhedo localizado na região sul do estado do Paraná. Com o objetivo de avaliar o efeito das podas mista e longa sobre a produção e qualidade da videira cv. Vênus na safra 2005/06. Foram utilizadas videiras com doze anos de idade, conduzidas em pérgula e espaçadas em 2,5 x 1,5 m. As videiras foram submetidas aos tratamentos de poda mista e longa. As variáveis avaliadas foram: porcentagem de gemas brotadas; número de cachos por planta; produção por planta; massa média dos cachos, sólidos solúveis (SS) e época de colheita. Observou-se que em relação à porcentagem de gemas brotadas, houve diferenças significativas entre as diferentes intensidades de poda, onde se obteve $87,93 \%$ de brotação na poda mista em relação à $76,68 \%$ na poda longa. A massa do cacho e o teor de sólidos solúveis não diferiram estatisticamente em ambas as podas. Em compensação, a produção dobrou com a poda longa em relação à poda mista, devido à poda longa produzir o dobro de cachos em relação à poda mista. A poda de frutificação invernal da videira 'Vênus' na região deve ser de intensidade média à longa deixandose por vara entre cinco a seis gemas para se obter maior número de cachos e que apresentem vigor adequado para a próxima poda de inverno. O tipo de poda influenciou significativamente na maturação das uvas, pois quando realizada a poda longa houve atraso da colheita em cinco dias.

Palavras-chave: condução de plantas, número de gemas, Vitisspp.

\begin{abstract}
A trial was conduced in a commercial vineyard located in a south region of Parana State. The vinegrape cv. Vênus with twelve years old spaced by $2.5 \times 1.5 \mathrm{~m}$ was used. The vinegrapes were submitted to mist and long pruned. The following variables were evaluated: percentage of sprouting; number of clusters; production; weigh medium of clusters, soluble solids (SS) and period of harvest. The percentage of buds pre-bloomed were different in mist prune and long prune treatments, the biggest medium was in mist prune $(87,93 \%)$ when compared which long prune $(76,68 \%)$. The weigh of clusters and the contents of soluble solids did not differ in both prunes. The production in plants submitted to long prune was twice as much as compared to mist prune, it was the double number of clusters when compared which the mist prune. The vinegrape 'Vênus' fructification prune in south area of Paraná State must be middle to long prune, with five or six buds per ramification, in other to obtain more clusters and show adequate vigor for the next winter prune. The prune influenced grapes ripening by delayed harvest for five days in long prune plants.
\end{abstract}

Key-words : plant conduction, number of buds, Vitisspp.

\footnotetext{
${ }^{1}$ Graduando em Agronomia da Universidade Estadual de Ponta Grossa (UEPG) - PR, bolsista PIBIC do CNPq. E-mail: marcellosozim@bol.com.br ${ }^{2}$ Eng. Agr., Dr. Prof. do Departamento de Fitotecnia e Fitossanidade da UEPG, Av. Gal. Carlos Cavalcante, 4748, 84030-900 Ponta Grossa, PR. Autor para correspondência. E-mail: rayub@uepg.br

${ }^{3}$ Eng. Agr., Dr, Pesquisador do IAPAR, Pólo de Ponta Grossa. E-mail: malgarim@yahoo.com
} 
SOZIM, M. et al. Efeito do tipo de poda...

INTRODUÇÃO

A videira é podada com o fim de equilibrar a frutificação e a vegetação (SOUSA, 1996). Em princípio, poda-se durante todo o período de repouso vegetativo, desde duas ou três semanas após a queda das folhas até a semana que precede a brotação, observando-se sempre os riscos de geadas tardias (CHAUVET e REYNIER, 1979). Nem todas as cultivares de videira se adaptam a qualquer método de poda. As possibilidades de adaptação estão relacionadas ao número de gemas férteis e à posição que estas ocupam ao longo do sarmento.

A intensidade da poda, definida pelo número de gemas a deixar por ramo da videira, depende da fertilidade das gemas, característica esta que pode ser definida como a capacidade de diferenciação de gemas vegetativas em frutíferas (HIDALGO, 1993). O número de gemas que permanecem nos sarmentos ou nos esporões após a poda de frutificação afeta diretamente a produção, mas podem ser afetadas por numerosas outras variáveis (WINKLER et al., 1974, DRY, 2000). ROBERTO et al. (2002) constataram diferenças significativas entre as podas curta, média e longa para a porcentagem de gemas brotadas e porcentagem de gemas brotadas com cacho, mostrando que o número de gemas deixado no sarmento ou esporão influencia nestas características da videira 'Niagara Rosada'. TONIETTO e CZERMAINSKI (1993), avaliando comportamento da videira 'Cabernet Franc', concluíram que os maiores índices de fertilidade foram encontrados da sexta à décima segunda gema ao longo da vara. CAMARGO et al. (1997) determinaram para a videira 'Perlette' cultivada no Vale do São Francisco que a poda curta afetou a produtividade pela baixa fertilidade das gemas basais. Nesta mesma região, uma vez que as videiras não passam por um período de dormência provocado por baixas temperaturas durante o inverno como nas regiões tradicionais, a posição da gema fértil na vara pode variar de um ciclo para outro (LEÃO, 1999). A fertilidade das gemas pode ser definida como a capacidade que apresentam para se diferenciar de vegetativas em frutíferas, podendo ser considerada como medida quantitativa do potencial de uma planta em produzir frutos. Dependendo da variedade há variação no setor do ramo em que são mais férteis (VALOR e BAUTISTA, 1997).

WINKLER et al. (1974) descreveram que o acúmulo de carboidratos nos ramos produtivos está estreitamente associada à formação da gema fértil e para muitas variedades, ocorre acumulo mais rápido de amido na porção mediana dos brotos e aí fica localizado o maior número de gemas frutíferas. Segundo estes autores, o acumulo de carboidratos parece atuar sobre a diferenciação floral pelo aumento da respiração ou pelo estímulo da síntese de substâncias nitrogenadas.

A cv. Vênus foi obtida pela Universidade do Arkansas, Estados Unidos e introduzida no Brasil pela Embrapa Uva e Vinho em 1984, apresentando, nas condições do Submédio São Francisco, características interessantes, destacando-se a precocidade, o tamanho de bagas, a boa fertilidade de gemas quando comparada a outras variedades sem sementes e produtividade média estimada de $24 \mathrm{t} \mathrm{ha}^{-1} \mathrm{ano}^{-1}$ (LEÃO, 1999). Entretanto na região Centro-Sul do Paraná, não há informações disponíveis acerca da intensidade de poda mais adequada para a sua produção e os resultados de outras regiões nem sempre podem ser extrapolados para essa região.

Neste contexto, realizou-se este trabalho com o objetivo de verificar o efeito das podas mista e longa na produção da videira cv. Vênus na região de Ponta Grossa, PR, na safra 2005/06.

\section{MATERIAL E MÉTODOS}

Este experimento foi realizado em vinhedo com doze anos de idade localizado no município de Ponta Grossa-PR, situado a $25^{\circ} 09 \mathrm{~S}$ e $50^{\circ} 00 \mathrm{O}$ e 849 metros de altitude em clima Cfb na classificação de Köeppen. Foram utilizadas videiras cv. Vênus

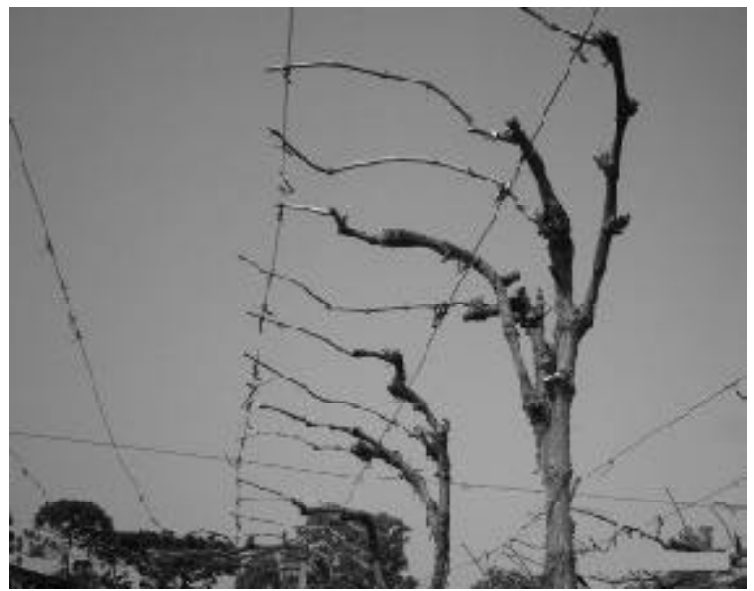

FIGURA 1 - Condução da videira cv. Vênus em a) poda mista (à esquerda) e b) poda longa (à direita). 
enxertadas sobre o porta-enxerto 420-A, no espaçamento $2,5 \times 1,5 \mathrm{~m}$ e conduzidas no sistema pérgula.

As videiras foram submetidas aos tratamentos de poda mista (três varas com quatro a oito gemas e esporões com uma a duas gemas), praticada pelo produtor, e poda longa (três varas com quatro a oito gemas e varas com cinco a seis gemas provenientes dos ramos mais velhos) na primeira semana de agosto, encontrando-se os ramos já maduros (Figura 1).

As variáveis avaliadas foram: porcentagem de brotação, número de cachos por planta, produção por planta, massa média dos cachos, sólidos solúveis (SS), e época de colheita.

O delineamento experimental foi inteiramente casualizado com quatro repetições e três plantas por parcela. Os dados experimentais foram submetidos à análise de variância para o esquema inteiramente casualizado e comparação de médias pelo teste de Tukey a $5 \%$ de probabilidade.

\section{RESULTADOS E DISCUSSÃO}

Em relação à porcentagem de gemas brotadas, observou-se que houve diferenças significativas entre as diferentes intensidades de poda, onde a maior média $87,93 \%$ foi obtida pela poda mista em relação aos $76,68 \%$ da poda longa. A massa do cacho e o teor de sólidos solúveis não foram afetados pelo tipo de poda, atingindo $0,15 \mathrm{~kg}$, um pouco abaixo do descrito para esta variedade, e $16,58 \stackrel{\circ}{\circ}$ Brix, respectivamente. Em compensação, a produção dobrou com a poda longa em relação à poda mista (Tabela 1), devido à poda longa ter produzido o dobro do número de cachos em relação à poda mista. O número de cachos constitui-se num dos principais componentes da produtividade e pode ser determinado pela poda e pela fertilidade das gemas.

TABELA 1 - Avaliação da porcentagem de gemas brotadas, massa do cacho, número de cachos por planta, produção da videira e sólidos solúveis da cv. Vênus em função do tipo de poda na safra 2005/06. Ponta Grossa, PR.

\begin{tabular}{lccccc}
\hline Tratamentos & $\begin{array}{c}\text { Gemas } \\
\text { brotadas (\%) }\end{array}$ & $\begin{array}{c}\text { Massa do cacho } \\
(\mathrm{kg})\end{array}$ & $\begin{array}{c}\text { №. de cachos } \\
\left(\text { planta }^{-1}\right)\end{array}$ & $\begin{array}{c}\text { Produção } \\
\left.\text { (kg planta }^{-1}\right)\end{array}$ & $\begin{array}{c}\text { Sólidos } \\
\text { solúveis } \\
(\text { Borix })\end{array}$ \\
\hline Poda Mista & $87,93 \mathrm{~A}$ & $0,15 \mathrm{~A}$ & $36,67 \mathrm{~B}$ & $5,47 \mathrm{~B}$ & $16,58 \mathrm{~A}$ \\
Poda Longa & $76,68 \mathrm{~B}$ & $0,14 \mathrm{~A}$ & $71,92 \mathrm{~A}$ & $10,31 \mathrm{~A}$ & $16,62 \mathrm{~A}$ \\
\hline
\end{tabular}

Médias seguidas de mesma letra, na coluna, não diferem entre si pelo teste de Tukey a 5\% de probabilidade.

As podas dos anos anteriores têm muita influência sobre a formação das gemas, quer frutífera quer vegetativa. As severas podas passadas, comumente praticadas na região, privaram as plantas de grande parte de sua copa. Como conseqüência, espera-se muita vegetação e pouco florescimento, o que está de acordo com SOUSA (1986). Este vigor induzido pela poda severa reduz a fertilidade das gemas, principalmente em cultivares apirenas (SHIKHAMANY, 1999).

Segundo ROBERTO et al. (2002), uma segunda época de poda na cv. Niagara Rosada no verão, com intensidade média à longa ( 4 a 8 gemas) favorece a uma maior porcentagem de gemas férteis, possivelmente devido ao maior acúmulo de carboidratos na porção mediana dos ramos nesta fase fenológica da planta, favorecendo uma maior porcentagem de gemas diferenciadas em gemas floríferas; diferindo assim significativamente da poda curta, na produção de uvas fora de época da videira 'Niagara' que apresentou limitações para a produção de gemas férteis na base do ramo. O conhecimento da posição das gemas férteis em cada variedade é importante na orientação da poda adotada no vinhedo, com conseqüentes aumentos em produtidade (VIEIRA et al., 2006). Segundo SANSAVI e FANIGLIUGO (1998) as videiras 'Centenial Seedless' e 'Sugraone' apresentaram maior fertilidade entre a $6^{\underline{a}}$ e a $18^{\mathrm{a}}$ gemas, com maiores produtividades em plantas submetidas a poda longa.

Os resultados obtidos neste trabalho evidenciam que a poda de inverno da videira 'Vênus' na região Centro-Sul do Paraná deve ser direcionada à poda de intensidade média à longa, deixando-se entre cinco a seis gemas por vara para se obter maior número de brotações que originem cachos e que apresentem vigor adequado para a próxima poda de inverno, mantendo o equilíbrio entre a vegetação e a frutificação das plantas. O que está de acordo com LEÃO e PEREIRA (2001) que chegaram à mesma conclusão trabalhando com a 'Venus' no Vale do Submédio do São Francisco.

O tipo de poda influenciou significativamente a maturação das uvas, pois quando se utilizou a poda mista, a colheita foi antecipada, enquanto que nas plantas podadas com maior número de gemas a colheita foi retardada em cinco dias, favorecendo um maior escalonamento da colheita e melhor uso dos fatores de produção na propriedade (Tabela 2). Este fato se deve a maior produção nas plantas com poda longa, que levaram a um amadurecimento tardio dos cachos. 
SOZIM, M. et al. Efeito do tipo de poda...

TABELA 2 - Efeito do tipo de poda na antecipação da colheita em kg por planta em videiras 'Vênus' na safra 2005/06, em Ponta Grossa, PR.

\begin{tabular}{lcccccc}
\hline Poda Colheita $^{(20 / 12 / 05)}$ & $(24 / 12 / 05)$ & $(29 / 12 / 05)$ & $(31 / 12 / 05)$ & $(05 / 01 / 06)$ & $(10 / 01 / 06)$ \\
\hline & & & & & \\
Poda curta & $1,0653 \mathrm{Aab}$ & $1,1475 \mathrm{Aab}$ & $1,3420 \mathrm{Aa}$ & $1,3470 \mathrm{Ba}$ & $0,5622 \mathrm{Bbc}$ & $0,0000 \mathrm{Bc}$ \\
Poda longa & $0,3538 \mathrm{Bd}$ & $0,5608 \mathrm{Bd}$ & $1,3848 \mathrm{Ac}$ & $2,2623 \mathrm{Ab}$ & $2,3023 \mathrm{Ab}$ & $3,4428 \mathrm{Aa}$
\end{tabular}

Médias seguidas de mesma letra maiúscula na coluna e minúscula na linha, não diferem entre si pelo teste de Tukey a $5 \%$ de probabilidade.

\section{CONCLUSÕES}

Videiras da cv. Vênus submetidas à poda longa com maior número de ramos produtivos apresentaram maior número de cachos por planta e maior produtividade.

A poda longa retardou a colheita em cinco dias.

\section{REFERÊNCIAS}

1. CAMARGO, U.A.; MASHIMA, C.H.; CZERMAINSKI, A.B.C. Avaliação de cultivares de uvas apirênicas no Vale do São Francisco. Bento Gonçalves: EMBRAPA-CNPUV, 1997. 8 p. EMBRAPA-CNPUV. (Comunicado Técnico, 26).

2. CHAUVET, M.; REYNIER, A. Manual de viticultura. 3 ed. Portugal: Narciso Correia, 1979. 312 p.

3. DRY, P. R. Canopy management for fruitfulness. Australian Journal of Grape and Wine Research, Adelaide, v. 6, n. 2, p. 109-115, 2000.

4. HIDALGO, L. Tratado de viticultura general. Madrid: Mundi Prensa, 1993. 690 p.

5. LEÃO, P.C.S. Avaliação do comportamento fenológico e produtivo de seis variedades de uva sem sementes no Vale do Rio São Francisco. Jaboticabal, 1999. 124 f. Dissertação (Mestrado em Agronomia) - Faculdade de Ciências Agrárias e Veterinárias, Universidade Estadual Paulista.

6. LEÃO, P.C.S.; PEREIRA, F.M. Estudo da brotação e da fertilidade de gemas de cultivares de uvas sem sementes nas condições tropicais do Vale submédio São Francisco. Revista Brasileira de Fruticultura, Jaboticabal, v. 23, n. 1, p. 30-34, 2001.

7. ROBERTO, S.R.; KANAI, H.T.; YANO, M.Y.; SASANO, E.M.; GENTA, W. Efeito da poda de frutificação sobre a fertilidade de gemas da videira 'Niagara Rosada' produzida fora de época no Paraná, 2002. In: CONGRESO BRASILEIRO DE FRUTICULTURA, 17., Belém, 2002. Anais. Belém: Sociedade Brasileira de Fruticultura, 2002. Disponível em: <http:// WWW.ufpel.edu.br/sbfruti/anais_xvii_cbf/fitotecnia/009.htm>. Acessado em 10 de Julho de 2005.

8. SANSAVINI, S.; FANIGLIUGO, G. Fertilita delle gemme e influenza della potatura sullafrutifica zione delle uve pirene 'Centennial Seedless' e 'Sugreone'. Rivista di Frutticoltura e di Ortofloricultura, Bolonha, v. 60, n. 2, p. 55-60, 1998.

9. SHIKHAMANY, S.D. Physiology and cultural practices to produce seedless grapes in tropical enviroments. In: CONGRESSO BRASILEIRO DE VITICULTURA E ENOLOGIA. Anais. Bento Gonçalves: Embrapa-CNPUV, 1999. p. 43-48.

10. SOUZA, J.S. I. Poda das plantas frutíferas. São Paulo: Nobel, 1986. 224 p.

11. SOUSA, J.S.I. Uvas para o Brasil. 2. ed. Piracicaba: FEALQ, 1996. 791 p.

12. TONIETTO, J.; CZERMAINSKI, A.B.C. Brotação e fertilidade das gemas da videira 'Cabernet Franc'. Revista Brasileira de Fruticultura, Jaboticabal, v. 15, n. 1, p. 185-192, 1993.

13. VALOR, O.; BAUTISTA, D. Brotacion y fertilidad de yemas en tres cultivares de vid para vino. Agronomia Tropical, Maracay, v. 47, n. 3, p. 347-358, 1997.

14. VIEIRA, C.R.Y.I.; PIRES, E.J.P.; TECCHIO, M.A.; OTSUBO, I.M.N.; VIEIRA, M.C.; YAMASAKI, A.K.; BORTOLANZA, O. Fertilidade de gemas de videiras 'Niagara Rosada' de acordo com o sistema de condução. Revista Brasileira de Fruticultura, Jaboticabal, v. 28, n. 1, p. 136-138, 2006.

15. WINKLER, A.J.; COOK, J.A.; KLIEWER, W.M.; LIDER, L.A. General viticulture. Berkeley: University of California Press, 1974. $710 \mathrm{p}$ 